\title{
Влияние зарядового состояния ионов ксенона на профиль распределения по глубине при имплантации в кремний
}

\author{
(C) Ю.В. Балакшин ${ }^{1,4}$, A.B. Кожемяко ${ }^{2}$, S. Petrovic ${ }^{3}$, M. Erich $^{3}$, A.A. Шемухин ${ }^{1,4}$, B.C. Черныш ${ }^{2}$ \\ ${ }^{1}$ Научно-исследовательский институт ядерной фризики им. Д.В. Скобельцына, \\ 119234 Москва, Россия \\ ${ }^{2}$ Московский государственноый университет им. М.В. Ломоносова (фризический фракультет), \\ 119991 Москва, Россия \\ ${ }^{3}$ Институт ядерных наук Винча, \\ 11351 Винча, Белград, Сербия \\ ${ }^{4}$ Центр квантовых технологий Московского государственного университета им. М.В. Ломоносова, \\ 119991 Москва, Россия \\ E-mail: balakshiny@gmail.com
}

Поступила в Редакцию 19 марта 2019 г.

В окончательной редакции 27 марта 2019 г.

Принята к публикации 1 апреля 2019 г.

\begin{abstract}
Представлены экспериментальные распределения концентрации имплантированных ионов ксенона по глубине в зависимости от их зарядового состояния и энергии облучения. Ионы ксенона в зарядовых состояниях $q=1-20$ и с энергиями в диапазоне от 50 до 400 кэВ были внедрены в монокристаллический кремний. Облучение проводилось в направлении, не совпадающем с кристаллографическими осями кристалла для исключения эффекта каналирования. Флюенс ионов варьировался в пределах $5 \cdot\left(10^{14}-10^{15}\right)$ ион $/ \mathrm{cm}^{2}$. Облучение однозарядными ионами и изучение образцов методом спектроскопии резерфордовского обратного рассеяния проводилось на ускорительном комплексе HVEЕ МГУ. Имплантация многозарядных ионов проводилась на ускорительном комплексе FAMA Института ядерных наук Винча. С помощью спектроскопии резерфордовского обратного рассеяния получены профили распределения по глубине внедренных ионов. Экспериментальные результаты сопоставлены с компьютерными расчетами. Показано, что средний проективный пробег многозарядных ионов в большинстве случаев имеет меньшие значения в сравнении со средним проективным пробегом однозарядных ионов и результатами компьютерного моделирования.
\end{abstract}

Ключевые слова: ионная имплантация, многозарядные ионы, спектроскопия резерфордовского обратного рассеяния (РОР).

DOI: $10.21883 /$ FTP.2019.08.47990.9108

\section{1. Введение}

С 50-х годов прошлого века началось изучение взаимодействия многозарядных ионов с поверхностью. Основной целью первых исследований было изучение вторичной электронной эмиссии с коллекторов для оценки влияния ионных потоков во вторичной масс-спектрометрии. В ряде экспериментальных и теоретических работ было показано, что ионно-индуцированная потенциальная эмиссия зависит от зарядового состояния падающих частиц. Установлено также, что определенный вклад в процессы эмиссии вносят атомное возбуждение, вид ионов и состояние поверхности. В качестве альтернативных направлений изучения взаимодействия многозарядных ионов с поверхностью рассматривались следующие: десорбция из межпланетных кластеров в астрофизике и космологии, спектроскопия атомов c незаполненными внутренними оболочками (hollow atoms) в атомной физике, устройства памяти высокой плотности, плазменные технологии, техники очистки поверхности в полупроводниковой промышленности, взаимодействие плазмы со стенками в термоядерных реакторах [1].
Разработка новых типов источников позволила получать ионы высокой зарядности, например, источник на электрон-циклотронном резонансе (ECR) или источник c ловушкой на электронном пучке (EBIT). Развитие ускорительной и вакуумной техники предоставило возможность проведения разнообразных экспериментальных работ в области взаимодействия многозарядных ионов с поверхностью. В большинстве работ, посвященных многозарядным ионам, изучались потенциальная электронная эмиссия с поверхности, распыление мишеней, рассеяние ионов, зарядовое равновесие в процессе прохождения ионов через тонкую мишень [2-6]. Было проведено большое количество работ, связанных с образованием треков под действием быстрых многозарядных ионов, их влияние на структуру и состав мишени, создание трековых мембран [7-10].

В ряде работ показано применение многозарядных ионов больших энергий для модификации свойств материалов. Например, под действием облучения многозарядными ионами неона было показано изменение магнитных свойств пленки феррита цинка [11]. Корреляция между упрочнением поверхности и структурной модификацией алюминиевых сплавов под действием пучка 
многозарядных ионов азота показана в работах [12,13]. В работе [14] показано формирование слоев $\beta$-силицида железа в кремнии $n$-типа под действием облучения многозарядными ионами железа, различные ориентации силицида железа были получены при различных зарядовых состояниях.

Применение многозарядных ионов малых энергий продемонстрировано в $[15,16]$ для получения ультратонких изолирующих слоев (0.3-2 нм) оксида кремния, однородных по составу и структуре, с малой шероховатостью поверхности. По аналогии с кластерными ионами $[17,18]$ применение медленных многозарядных ионов позволяет модифицировать приповерхностные слои облучаемых мишеней без ионно-индуцированного дефектообразования на глубине.

Воздействие многозарядных ионов на поверхность может быть использовано в качестве техники наноструктурирования. В работе, посвященной модификации поверхности сапфира, было показано, что облучение ионами ксенона в высоком зарядовом состоянии приводит к образованию наноструктур типа нанохиллоков (nanohillocks) [19]. Наблюдаемые результаты объяснялись величинами электронных потерь и передачей потенциальной энергии. В ряде работ было показано, что тип образующихся наноструктур (hillocks, кратеры, треки) и их размер зависят как от материала мишени, так и от параметров пучка, в частности зарядового состояния ионов и их кинетической энергии [20-23]. Касательно экспериментального изучения проникновения в мишени многозарядных ионов средних энергий проведено небольшое количество работ, оценивающих потери энергии и распределения по глубине внедряемых частиц [24].

Проведение детальных исследований по изучению профилей распределения по глубине имплантированных ионов в различных зарядовых состояниях, в частности сравнение с однозарядными ионами, позволит получить дополнительную информацию о взаимодействии многозарядных ионов с твердым телом. Продемонстрированное влияние зарядового состояния ионов на потери энергии было аналогично потерям энергии, наблюдаемым при облучении углеродных мембран [25], где наблюдалась квадратичная зависимость потерь энергии от зарядового состояния при облучении медленными ионами ксенона. Авторы работы предположили, что увеличение заряда иона приводит к увеличению потерь энергии посредством изменения величины потенциала упругого рассеяния. В работе [26] было показано влияние зарядового состояния иона на дефектообразование в графене. Авторы предположили, что в случае быстрых тяжелых однозарядных ионов энергия возбуждения электронной системы объемного материала передается кристаллической решетке и происходит локальный нагрев и отжиг дефектов. В случае облучения многозарядными ионами, по мнению авторов, данный эффект не так ярко выражен.
В данной работе показано влияние зарядового состояния ионов на параметры распределения по глубине в диапазоне энергий 50-400 кэВ в сравнении с однозарядными ионами и моделями, полученными в программах SRIM и MARLOWE. В качестве внедряемых ионов были выбраны ионы ксенона $\mathrm{Xe}^{131}$, исключающие химическое взаимодействие с мишенью, а также имеющие значительную массу для удобства в изучении методом резерфордовского обратного рассеяния (POP). В качестве мишени использовался монокристаллический кремний, так как данный материал является одним из наиболее применяемых и изученных в полупроводниковой технологии.

\section{2. Экспериментальные методы}

Ионами ксенона с энергией от 50 до 400 кэВ в различных зарядовых состояниях $(q=1-20)$ были облучены монокристаллические образцы кремния площадью $1 \times 1 \mathrm{~cm}^{2}$ с ориентацией (110). Облучение проводилось в направлении, не совпадающем с кристаллографическими осями кристалла (под углом $7^{\circ}$ по отношению к нормали к поверхности образца), во избежание эффекта каналирования ускоренных частиц в кристалле. Облучение проводилось при комнатной температуре, мощность пучка поддерживалась на исключающем термический отжиг уровне. Флюенсы облучения в различных случаях составляли $5 \cdot 10^{14}$ или $5 \cdot 10^{15}$ ион $/ \mathrm{cm}^{2}$. Облучение однозарядными ионами проводилось на ускорительном комплексе HVEE Московского университета, одна из линий которого приспособлена для контролируемого равномерного облучения твердотельных мишеней $[27,28]$. Однородность облучения обеспечивалась сканированием образца пучком в двух взаимно перпендикулярных направлениях с большой частотой. Система цилиндров Фарадея с подавлением вторичной электронной эмиссии использовалась для точного определения флюенса. Вакуум в камере во время облучения составлял $6 \cdot 10^{-7}$ мбар. Облучение многозарядными ионами проводилось в Институте ядерных наук Винча на ускорительном комплексе FAMA [29,30]. В процессе облучения образец сканировался под пучком для достижения однородности флюенса. Ток пучка ионов был в пределах 0.6-1 мкА. Давление в экспериментальной камере было на уровне $10^{-6}$ мбар.

Анализ образцов методом резерфордовского обратного рассеяния (РОР) проводился с применением ионов гелия при энергии 350 кэВ, что позволяет получать значительно больший сигнал от малых концентраций примесных атомов в сравнении с энергиями порядка мегаэлектронвольта за счет большего сечения упругого рассеяния. При этом отклонение сечения рассеяния от резерфордовского за счет экранирования ядра электронными оболочками для указанных параметров пучка и выбранных элементов незначительно. Диаметр пучка, падающего на исследуемый образец, составлял 1 мм, 


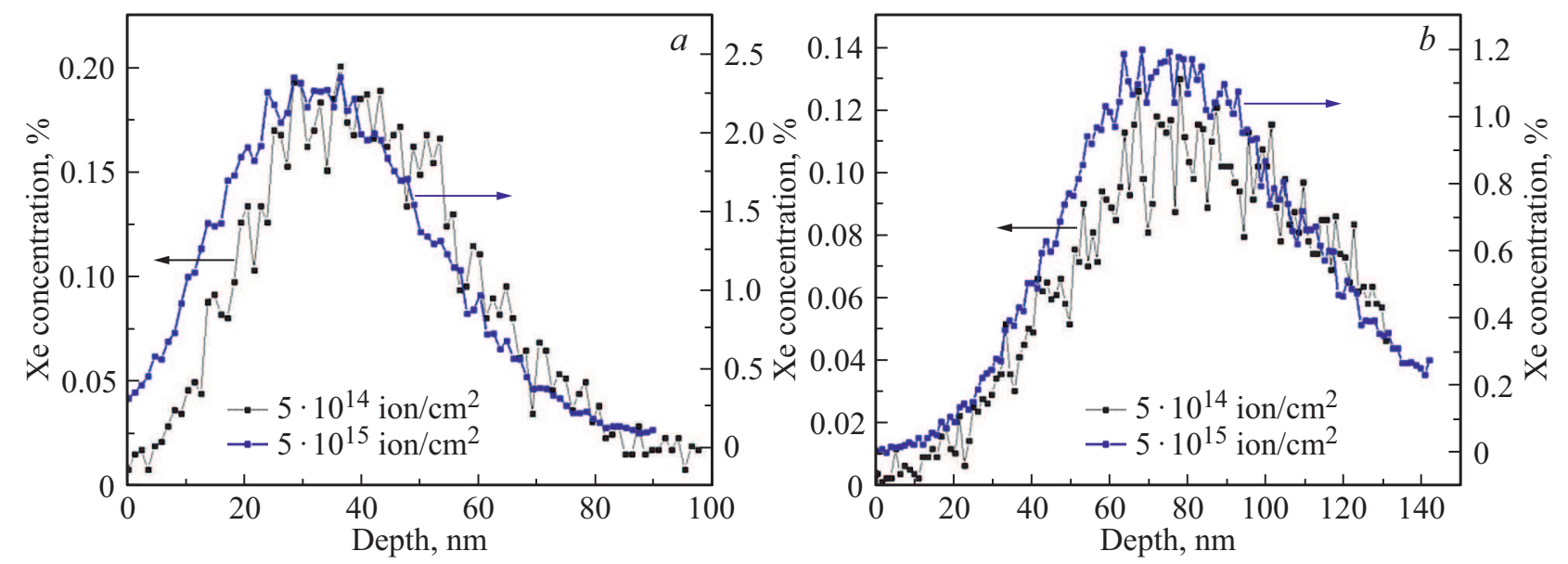

Рис. 1. Профили распределения по глубине ионов $\mathrm{Xe}^{+}$с энергией $100(a)$ и 200 кэВ $(b)$ для флюенсов $5 \cdot 10^{14}$ и $5 \cdot 10^{15}$ ион/см².

угловое геометрическое расхождение пучка составляло $0.12^{\circ}$. Спектры РОР были получены с применением кремниевого детектора поверхностно-барьерного типа диаметром 1 см, находящегося на удалении от мишени в 6 см, при угле рассеяния $160^{\circ}$. Флюенс анализирующих частиц контролировался системой мониторирования, основанной на получении спектров рассеяния пучка на вращающейся рамке, покрытой пленкой золота. Подробно линия РОР и спектрометрический тракт описаны в работе [31].

Используемые в дальнейших расчетах спектры РОР были получены в „random“ режиме, когда направление анализирующего пучка не совпадает с направлением кристаллографических осей в кристалле. Однако сравнение профилей распределения ксенона по глубине в „random“ и каналированном режимах было проведено и показало совпадение профилей, т.е. отсутствие замещения атомов кремния ионами ксенона в узлах решетки. Компьютерное моделирование профилей распределения ксенона по глубине было проведено с применением метода Монте-Карло для парных столкновений между налетающими ионами и изначально покоящимися атомами мишени. Были использованы программа SRIM [32] с применением ZBL потенциала и программа MARLOWE $[33,34]$ с применением потенциала Мольера. Моделирование при различных потенциалах показало близкие результаты распределения имплантированных ионов по глубине.

Исследования процессов распыления поверхности многозарядными ионами проводились не так интенсивно в сравнении с однозарядными ионами. Поэтому для оценки распыления под действием облучения многозарядными ионами граница раздела облученной и исходной области образца исследовалась методами атомно-силовой микроскопии с помощью прибоpa NT-MDT NTEGRA Spectra c применением полуконтактного метода измерения в области площадью $35 \times 35$ мкм.

\section{3. Результаты и обсуждение}

На рис. 1 представлены, полученные из спектров РОР, профили распределения по глубине внедренных с энергией 100 и 200 кэВ однозарядных ионов ксенона при флюенсах облучения $5 \cdot 10^{14}$ или $5 \cdot 10^{15}$ ион/см ${ }^{2}$. В ряде работ наблюдается зависимость формы профиля распределения примеси от флюенса облучения [35], что будет проверено для указанных параметров эксперимента. Отметим, что левая ось ординат на рис. 1, $a$ и $b$ соответствует меньшему флюенсу, правая ось ординат - большему. Согласно рис. 1, профиль распределения смещается к поверхности с ростом флюенса облучения для обеих энергий пучка, в то время как форма распределений в хвостовой части профилей с ростом флюенса меняется незначительно.

Согласно экспериментальным данным, представленным на рис. 1, средние проективные пробеги внедренных ионов с энергией 100 кэВ составили 34 и 29 нм для флюенсов $5 \cdot 10^{14}$ и $5 \cdot 10^{15}$ ион $/ \mathrm{cm}^{2}$ соответственно. Для энергии 200 кэВ средние проективные пробеги составляют 77 и 73 нм для флюенсов $5 \cdot 10^{14}$ и $5 \cdot 10^{15}$ ион $/ \mathrm{cm}^{2}$ соответственно. Расстояния между передними фронтами профилей для различных флюенсов на середине высоты профиля составляют 6 и 4 нм для энергий 100 и 200 кэВ соответственно, что согласуется с различием величин средних проективных пробегов. Для указанных флюенсов была оценена толщина распыленного слоя кремния под действием ионного облучения $X \propto Y \cdot F l$, где $X$ - толщина распыленного слоя, $Y$ - коэффициент распыления, $F l$ - флюенс облучения [36,37]. Коэффициент распыления атомов кремния ионами ксенона был взят согласно [38]. Согласно проведенным оценкам, для энергий 100 и 200 кэВ толщина распыленного слоя кремния при флюенсе $5 \cdot 10^{15}$ ион/см ${ }^{2}$ превышает толщину распыленного слоя при флюенсе $5 \cdot 10^{14}$ ион $/ \mathrm{cm}^{2}$ на величину $\sim 4$ нм. Эта величина соответствует наблюдаемому на рис. 1 смещению средних проективных пробегов и 

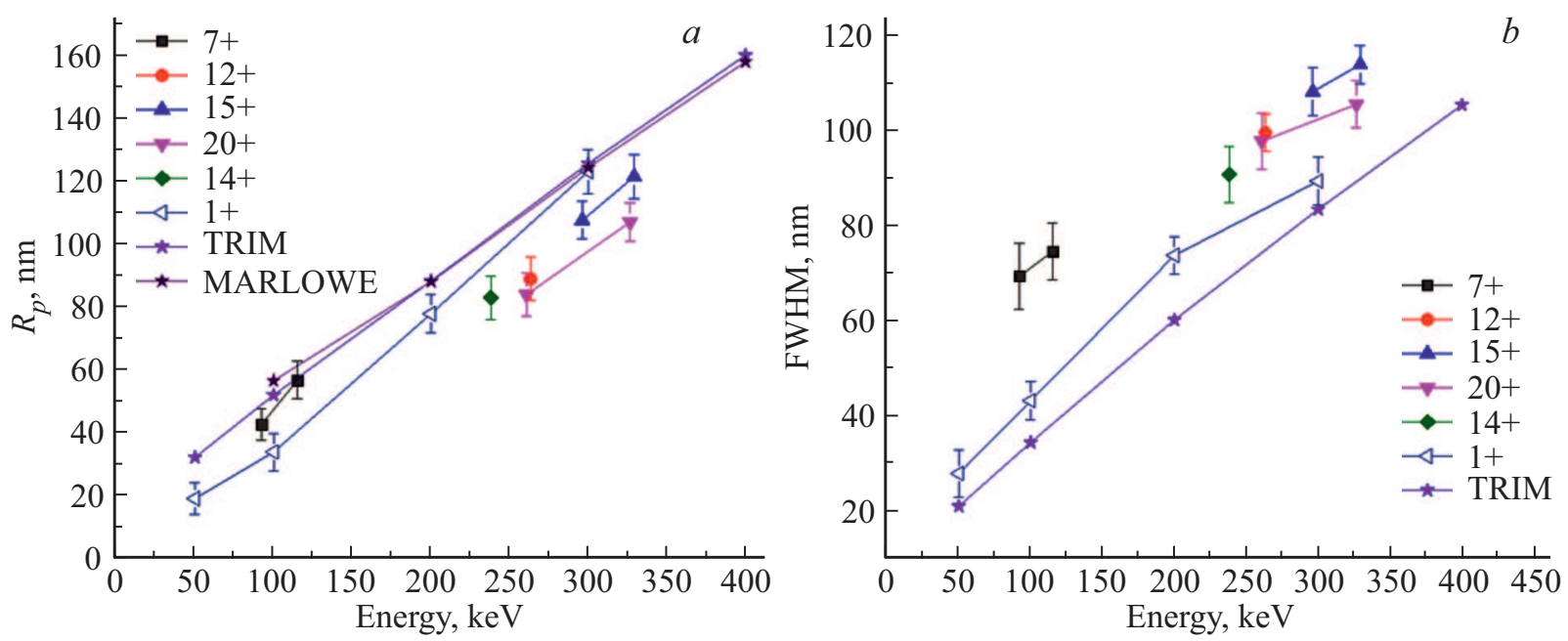

Рис. 2. Зависимость величин $R_{p}(a)$ и FWHM $(b)$ ионов ксенона от энергии в различных зарядовых состояниях.

передних фронтов профилей распределения по глубине, полученных при различных флюенсах облучения.

Согласно вышесказанному, дальнейшее облучение кремниевых образцов проводилось с флюенсом $5 \cdot 10^{15}$ ион $/ \mathrm{cm}^{2}$, что позволило значительно увеличить сигнал в процессе РОР анализа и уменьшить статистический разброс. Для образцов, облученных однозарядными ионами, при преобразовании спектров РОР в профили распределения по глубине было учтено смещение профилей за счет распыления поверхности образца. Для многозарядных ионов толщина распыленного слоя оценивалась методами атомно-силовой микроскопии.

Зависимость параметров профилей распределения имплантированных ионов по глубине от энергии для различных зарядовых состояний представлена на рис. 2. Согласно полученным экспериментальным результатам (см. рис. $2, a)$, средний проективный пробег $R_{p}$ однозарядных ионов ксенона в области энергий $50-200$ кэВ имеет меньшие значения в сравнении с результатами компьютерного моделирования в программах SRIM и MARLOWE, однако различие уменьшается с увеличением энергии и при энергии 300 кэВ экспериментальные и рассчитанные величины совпадают. Аналогичный результат наблюдался в работах [39-41].

Для профилей распределения многозарядных ионов (рис. 2,a) можно выделить две области: низкоэнергетичную и низкозарядовую область 1 и область 2 , где энергии более 220 кэВ и зарядовые состояния превышают $7+$. В случае зарядового состояния $7+$ и энергий вблизи 100 кэВ (область 1) наблюдается незначительное отличие экспериментальных средних проективных пробегов от результатов моделирования, при этом величина $R_{p}$ превышает значения, полученные для однозарядных ионов. Для области 2 наблюдается меньшее значение среднего проективного пробега многозарядных ионов ксенона по сравнению с расчетными и экспериментальными величинами для однозарядных ионов.
Отметим, что в области больших энергий (область 2), облучение проводилось ионами в относительно больших зарядовых состояниях $(12+, 14+, 15+, 20+)$. Отметим, что программы SRIM и MARLOWE не учитывают зарядовое состояние иона при его прохождении через твердое тело.

Отличие средних проективных пробегов между однои многозарядными ионами говорит о влиянии на величину $R_{p}$ не только энергии облучения, но и зарядового состояния, при этом отличие от расчетных величин $R_{p}$ с увеличением энергии и зарядового состояния возрастает. Известно, что профили распределения внедренной примеси определяются как упругими, так и неупругими потерями энергии. Возможно, наблюдаемое отличие средних проективных пробегов связано с различием неупругих потерь энергии. Другими словами, уменьшение величины $R_{p}$ может являться следствием увеличения общих потерь энергии и увеличения неупругой компоненты потерь в частности.

В то же время увеличение неупругих потерь энергии должно приводить к уширению профиля распределения примеси по глубине, что наблюдается на рис. $2, b$. Возрастание потерь энергии ускоренных частиц с увеличением заряда ускоряемых ионов при прохождении через углеродные пленки наблюдалось в работах $[42,43]$ : увеличение средних потерь энергии как функции заряда иона наблюдалось для $\mathrm{Xe}^{q+}$ и $\mathrm{Au}^{q+}$ в случае $q>40$, наблюдаемые результаты объяснялись вкладом зарядового обмена иона с твердым телом на процесс передачи энергии через конечное время снятия возбуждения (de-exitation) многозарядного иона. Отметим, что, согласно рис. $2, b$, в то время как величина FWHM профиля распределения для однозарядных ионов находится вблизи рассчитанных в SRIM значений, для многозарядных ионов наблюдается значительное отличие, при этом для зарядового состояния $7+$ наблюдается наибольшее отклонение. Аналогичное отличие величины 

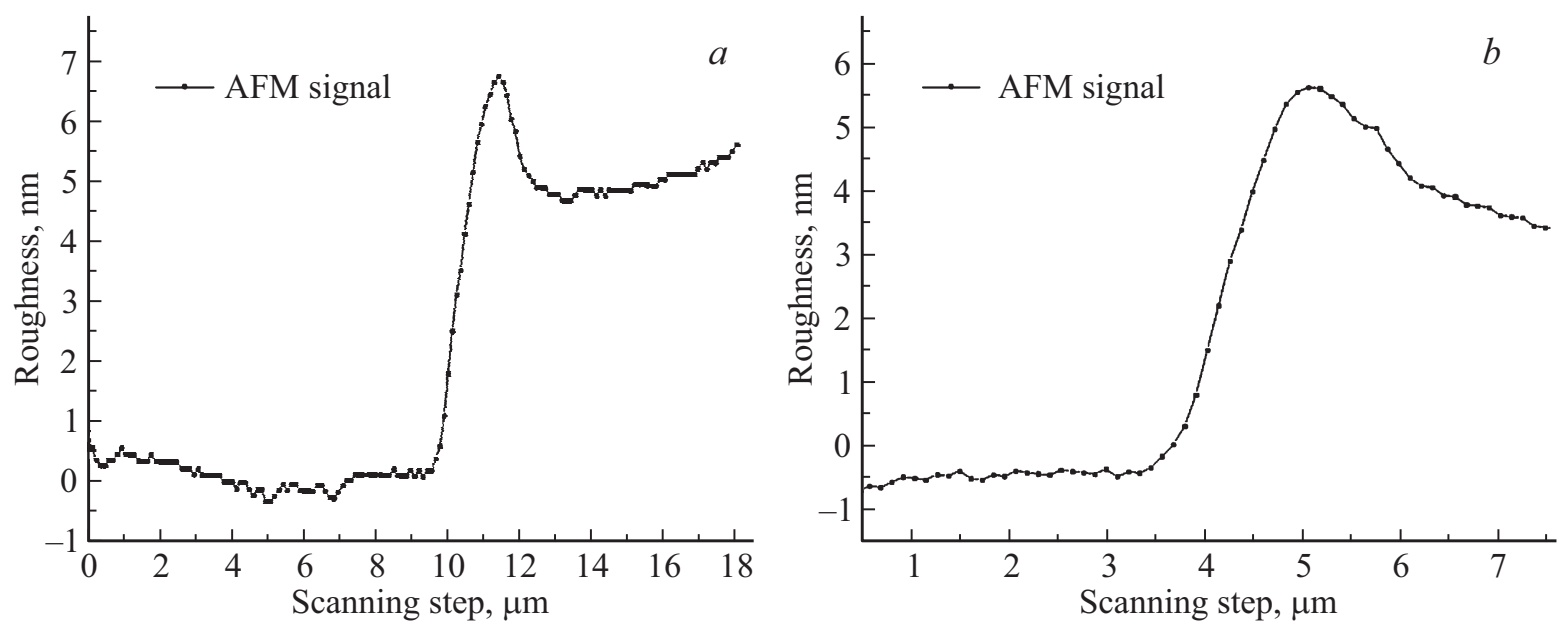

Рис. 3. Шероховатость границы раздела облученной и необлученной областей для различных зарядовых состояний: $q=12+(a)$ и $q=7+(b)$.

FWHM профиля распределения примеси, полученной при компьютерном моделировании и в результате облучения однозарядными ионами ксенона мишеней из кремния и оксида кремния, было продемонстрировано в работе [44].

В ряде работ показано, что увеличение зарядового состояния иона приводит к увеличению коэффициента распыления при облучении мишени [45-47]. Это явление связывают с увеличением распыления за счет большого потенциала между налетающим многозарядным ионом и поверхностью мишени, описывается эффект моделями кулоновского взрыва и опосредованного распыления дефектов (defect-mediated sputtering) [48,49]. Однако этот эффект наблюдается не для всех типов мишеней. В частности, для кремния, облучаемого ионами $\mathrm{Ar}^{q+}$ до значения зарядового состояния 9+, наблюдается кинетическое распыление [50], затем проявляется зависимость распыления от заряда иона. Общая тенденция эффекта в том, что с ростом зарядового состояния возрастает коэффициент распыления и разница может достигать порядка.

Полученные экспериментальные результаты, в частности меньшие значения средних проективных пробегов ионов (см. рис. 2,a), при зарядовых состояниях $12+, 14+, 15+, 20+$ в сравнении с однозарядными ионами возможно было бы объяснить значительным увеличением распыления поверхности за счет потенциального взаимодействия.

Измерения, проведенные с помощью атомно-силовой микроскопии для зарядовых состояний $12+$ и $7+$, представлены на рис. 3. Разность высот на границе облученной и необлученной областей имеет величину $\sim 5 \mathrm{Hм}$, что соответствует толщинам распыленного слоя под действием облучения однозарядными ионами. Пик, соответствующий самой границе раздела областей, превышает высоту исходного образца, но это является результатом несовершенства применяемого полуконтактного метода.

\section{4. Заключение}

Профили распределения по глубине одно- и многозарядных ионов были изучены в серии экспериментов по имплантации в диапазоне энергий 50-400 кэВ. Измерения проводились методом спектроскопии резерфордовского обратного рассеяния и сравнивались с результатами компьютерного моделирования в программах SRIM и MARLOWE с использованием потенциалов ZBL и Мольера соответственно. Было обнаружено отклонение в меньшую сторону средних проективных пробегов многозарядных ионов в области энергий 250-350 кэВ по отношению к пробегам однозарядных ионов, в то время как при энергии вблизи 100 кэВ величины пробегов многозарядных ионов приближены к рассчитанным и превышают пробеги однозарядных ионов. Было показано, что средние проективные пробеги для однозарядных ионов, полученные в результате моделирования, в области энергий 50-250 кэВ превышают экспериментальные значения, но с увеличением энергии до 300 кэВ пробеги совпадают. Для величин ширины профиля распределения на полувысоте наблюдается иная картина. FWHM многозарядных ионов значительно превышают экспериментальные и рассчитанные величины, соответствующие однозарядным ионам. При этом наблюдается уменьшение отклонения величин FWHM многозарядных ионов от однозарядных при увеличении энергии облучения. Посредством применения атомно-силовой микроскопии на границе раздела облученной и сходной областей образца было показано, что в случае применения многозарядных ионов ксенона в различных зарядовых состояниях коэффициент распыления имеет значения, близкие к значениям, характерным для однозарядных ионов.

Результаты проведенных экспериментов демонстрируют некоторые особенности распределения по глубине многозарядных ионов при прохождении через вещество. Если в случае однозарядных ионов профили распреде- 
ления по глубине определяются упругими и неупругими потерями энергии в отсутствие значительной модификации структуры и состава мишени (т.е. при небольших флюенсах), закономерности проникновения многозарядных ионов в вещество имеют дополнительные факторы, связанные с зарядовым состоянием налетающих частиц. Важную роль приобретают процессы перезарядки, т.е. обмена зарядом между налетающими частицами и атомами мишени. Нейтрализация избыточного заряда ускоренных частиц в поверхностных слоях мишени не объясняет представленные в работе особенности распределения внедренных ионов.

Эффекты от взаимодействия многозарядных ионов с мишенью предполагают применение в различных областях техники. Для контролируемого и предсказуемого применения пучков многозарядных ионов требуется понимание влияния особенностей кинетического и потенциального взаимодействия, потерь энергии и изменения зарядового состояния в процессе проникновения. Поэтому требуется более детальное дальнейшее исследование этих процессов.

\section{Финансирование работы}

Исследование проведено при финансовой поддержке РФФИ в рамках научного проекта № 18-32-00833мол_а. M. Erich и S. Petrovic отмечают поддержку данной работы Министерством образования, науки и технологического развития Сербии в рамках проекта „Физика и химия ионных пучков“" № III 45006, в частности при проведении работ на ускорительном комплексе FAMA.

\section{Благодарности}

Авторы работы выражают благодарность персоналу ускорительного комплекса FAMA за проведение экспериментов по облучению многозарядными ионами.

\section{Конфликт интересов}

Авторы заявляют, что у них нет конфликта интересов.

\section{Список литературы}

[1] A. Arnau, F. Aurmayr, P.M. Echenique, M. Grether, W. Heiland, J. Limburg, R. Morgenstern, P. Roncin, S. Schippers, R. Schuch, N. Stolterfoht, P. Varga, T.J.M. Zouros, H.P. Winter. Surf. Sci. Rep., 27, 113 (1997).

[2] T. Schenkel, A.V. Hamza, A.V. Barnes, D.H. Schneider. Progr. Surf. Sci., 61, 23 (1999).

[3] F. Aumayr, H.P. Winter. Nucl. Instr. Meth. Phys. Res. B, 233, 111 (2005).

[4] R.A. Wilhelm, E. Gruber, J. Schwestka, R. Kozubek, T.I. Madeira, J.P. Marques, J. Kobus, A.V. Krasheninnikov, M. Schleberger, F. Aumayr. Phys. Rev. Lett., 119, 103410 (2017).

[5] N.V. Novikov, Ya.A. Teplova. Phys. Lett. A, 378, 1286 (2014).

[6] Yu.A. Belkova, N.V. Novikov, Ya.A. Teplova. Nucl. Instr. Meth. Phys. Res. B, 343, 110 (2015).
[7] V.A. Skuratov, J. O’Connell, A.S. Sohatsky, J. Neethling. Nucl. Instr. Meth. Phys. Res. B, 327, 89 (2014).

[8] E.G. Njoroge, C.C. Theron, J.B. Malherbe, N.G. Van der Berg, T.T. Hlatshwayo, V.A. Skuratov. Nucl. Instr. Meth. Phys. Res. B, 354, 249 (2015).

[9] E.Yu Kaniukov, J. Ustarroz, D. Yakimchuk, M.N. Petrova, H.A. Terryn, V. Sivakov, A.V. Petrov. Nanotechnology, 27, 115305 (2016).

[10] Y. Chen, Z. Zhao, J. Dai, Y. Liu, H. Ma, R. Nie. Rad. Measurements, 43, s111 (2008).

[11] E.V. Gafton, G. Bulai, O.F. Caltun, S. Cervera, S. Mace, M. Trassinelli, S. Steydli, D. Vernhet. Appl. Surf. Sci., 33036, 1 (2016).

[12] E. Hug, S. Thibault, D. Chateigner, L. Maunoury. Surf. Coat. Techn., 206, 5028 (2012).

[13] S. Thibault, E. Hug. Appl. Surf. Sci., 310, 311 (2014).

[14] M. Tomida, Y. Kato, T. Asaji. Nucl. Instr. Meth. Phys. Res. B, 237, 83 (2005).

[15] G. Borsoni, N. Bechu, M. Gros-Jean, M.L. Korwin-Pawlowski, R. Laffitte, V.Le Roux, L. Vallier, N. Rochat, C. Wyon. Microelectronics Reliability, 41, 1063 (2001).

[16] G. Borsoni, V. Le Roux, R. Laffitte, S. Kerdiles, N. Bechu, L. Vallier, M.L. Korwin-Pawlowski, C. Vannuffel, F. Bertin, C. Vergnaud, A. Chabli, C. Wyon. Solid-State Electron., 46, 1855 (2002).

[17] A.E. Ieshkin, S.E. Svyakhovskiy, V.S. Chernysh. Vacuum, 148, 272 (2018).

[18] A.E. Ieshkin, D.S. Kireev, Yu.A. Ermakov, A.S. Trifonov, D.E. Presnov, A.V. Garshev, Yu.V. Anufriev, I.G. Prokhorova, V.A. Krupenin, V.S. Chernysh. Nucl. Instr. Meth. Phys. Res. B, 421, 27 (2018).

[19] A.S. El-Said, R.A. Wilhelm, R. Heller, S. Akhmadaliev, S. Fasco. Nucl. Instr. Meth. Phys. Res. B, 317, 170 (2013).

[20] A.S. El-Said, R. Heller, S. Facsko. Nucl. Instr. Meth. Phys. Res. B, 269, 901 (2011).

[21] R. Heller, S. Facsko, R.A. Wilhelm, W. Möller. Phys. Rev. Lett., 101, 096102 (2009).

[22] M. Tona, H. Watanabe, S. Takahashi, N. Nakamura, N. Yoshiyasu, N.M. Sakurai, T. Terui, S. Mashiko, C. Yamada, S. Ohtani. Surf. Sci., 601, 723 (2007).

[23] B.E. O’Rourke, M. Flores, V.A. Esaulov, Y. Yamazaki. Nucl. Instr. Meth. Phys. Res. B, 229, 68 (2013).

[24] J.P. Biersack. Nucl. Instr. Meth. Phys. Res. B, 80/81, 12 (1993).

[25] R.A. Wilhelm, E. Gruber, R. Ritter, R. Heller, S. Facsko, F. Aumayr. Phys. Rev. Lett., 112 (15), 1 (2014).

[26] P. Ernst, R. Kozubek, L. Madaub, J. Sonntag, A. Lorke, M. Schleberger. Nucl. Instr. Meth. Phys. Res. B, 382, 71 (2016).

[27] A.A. Shemukhin, A.V. Nazarov, Yu.V. Balakshin, V.S. Chernysh, Nucl. Instr. Meth. Phys. Res. B, 354, 274 (2015).

[28] А.А. Шемухин, Ю.В. Балакшин, В.С. Черныш, С.А. Голубков, Н.Н. Егоров, А.И. Сидоров. ФТП, 48 (4), 535 (2014).

[29] M. Erich, M. Kokkoris, S. Fazinic, S. Petrovic. Nucl. Instr. Meth. Phys. Res. B, 381, 96 (2016).

[30] M. Erich, M. Kokkoris, S. Fazinic, S. Petrovic. Nucl. Instr. Meth. Phys. Res. B, 416, 89 (2018).

[31] Ю.В. Балакшин, А.А. Шемухин, А.В. Назаров, А.В. Кожемяко, В.С. Черныш. ЖТФ, 88 (12), 1900 (2018).

[32] J.F. Ziegler, M.D. Ziegler, J.P. Biersack. Nucl. Instr. Meth. Phys. Res. B, 268, 1818 (2010). 
[33] M.T. Robinson. Nucl. Instr. Meth. Phys. Res. B, 48, 408 (1990).

[34] M.T. Robinson. Nucl. Instr. Meth. Phys. Res. B, 67, 396 (1992).

[35] P. Philipp, T. Wirtz, H.-N. Migeon, H. Scherrer. Int. J. Mass Spectrometry, 261, 91 (2007).

[36] P. Sigmund. Nucl. Instr. Meth. Phys. Res. B, 27 (1), 1 (1987).

[37] P. Sigmund. Nucl. Instr. Meth. Phys. Res. B, 406, 391 (2017).

[38] Y. Yamamura, H. Tawara. NIFS-DATA, 23, 29 (1995).

[39] J.F. Ziegler. Nucl. Instr. Meth. Phys. Res. B, 6, 272 (1985).

[40] А.В. Кожемяко, Ю.В. Балакшин, А.А. Шемухин, В.С. Черныш. ФТП, 51 (6), 778 (2017).

[41] A.A. Shemukhin, A.V. Kozhemiako, Yu.V. Balakshin, V.S. Chernysh. IOP Conf. Ser.: J. Physics: Conf. Ser., 917, 1 (2017).

[42] T. Schenkel, M.A. Briere, A.V. Barnes, A.V. Hamza, K. Bethge, H. Schmidt-Bocking, D.H. Schneider. Phys. Rev. Lett., 79 (11), 2030 (1997).

[43] T. Schenkel, A.V. Hamza, A.V. Barnes, D.H. Schneider. Phys. Rev. A, 56 (3), 1701 (1997).

[44] M. Behar, P.F.P. Fichter, P.L. Grande, F.C. Zawislak. Mater. Sci. Engin., R15, 1 (1995).

[45] F. Aumayr, P. Varga, H.P. Winter. Int. J. Mass Spectrometry, 192, 415 (1999).

[46] B.L. Oksengendler, F.G. Djurabekova, S.E. Maksimov, N.Yu. Turaev, N.N. Turaeva. Vacuum, 105, 70 (2014).

[47] S. Meyer, A. Wucher. Nucl. Instr. Meth. Phys. Res. B, 267, 646 (2009).

[48] R. Gonzalez-Arrabal, N. Gordillo, G. Garcia, D.O. Boerma, V.A. Khodyrev. Nucl. Instr. Meth. Phys. Res. B, $249(1-2)$, 65 (2006).

[49] V.A. Khodyrev, V.S. Kulikauskas, C. Yang. Nucl. Instr. Meth. Phys. Res. B, 195 (3-4), 259 (2002).

[50] P. Varga, T. Neidhart, M. Sporn, G. Libiseller, M. Schmid, F. Aumayr, H.P. Winter. Physica Scripta, T73, 307 (1997).

\section{Influence of xenon ions charge state on depth distribution profile during implantation in silicon}

Yu.V. Balakshin 1,4 A.V. Kozhemiako ${ }^{2}$, S. Petrovic ${ }^{3}$, M. Erich ${ }^{3}$, A.A. Shemukhin ${ }^{1,4}$, V.S. Chernysh ${ }^{2}$

\author{
${ }^{1}$ Skobeltsyn Institute of Nuclear Physics, \\ Lomonosov Moscow State University, \\ 119234 Moscow, Russia \\ ${ }^{2}$ Faculty of Physics, \\ Lomonosov Moscow State University, \\ 119991 Moscow, Russia \\ ${ }^{3}$ Vinča Institute of Nuclear Sciences, \\ 11351 Vinča, Belgrad, Serbia \\ ${ }^{4}$ Center for quantum technologies, \\ Lomonosov Moscow State University, \\ 119991 Moscow, Russia
}

\begin{abstract}
The depth distributions of implanted Xe ions were measured depending on their charge state and energy. Xenon ions with various charge states $(q=1-20)$ and energies from 50 to $400 \mathrm{keV}$ were implanted in silicon single crystal. Irradiations were carried out in random direction to avoid channeling effect. Implantation fluences varied from $5 \cdot\left(10^{14}-10^{15}\right) \mathrm{ion} / \mathrm{cm}^{2}$. Single-charged ion implantations and RBS analysis were carried out at MSU HVEE implanter. Irradiations by multi-charged ions were performed at FAMA, Vinča Institute of Nuclear Sciences. Depth profiles of implanted ions were obtained by using Rutherford backscattering technique. Experimental results are compared with computer simulations. In most cases the average projected ranges of multi-charged ions were found to have smaller values compare to the projectiles for single-charged ions and theoretical predictions.
\end{abstract}

